\title{
Dados de Ocorrência de Infecção Hospitalar, da Mortalidade por Causas Externas e da Leishmaniose Visceral Americana no Estado de São Paulo
}

\author{
Data on hospital infection, external causes of death, and visceral leishmaniose \\ in the state of São Paulo, Brazil
}

Secretaria de Estado da Saúde de São Paulo*

\section{INVESTIGAÇÃO EPIDEMIOLÓGICA DE SURTO DE INFECÇÃO HOSPITALAR EM UNIDADE DE TERAPIA INTENSIVA NEONATAL - ITAQUAQUECETUBA, SÃO PAULO}

Entre os dias 9 e 16 de outubro de 2003, ocorreram nove óbitos de pacientes atendidos na UTI neonatal do Hospital Santa Marcelina de Itaquaquecetuba. Esses casos, que representavam a totalidade de neonatos internados na unidade, foram notificados às autoridades estaduais de saúde em 16 de outubro. Como medida de cautela, a UTI foi temporariamente desativada. Investigações foram conduzidas pelas vigilâncias (epidemiológica e sanitária) em suas instâncias locais (Direção Regional de Saúde - Dir) e centrais. Técnicos da Divisão de Infecção Hospitalar, do Centro de Vigilância Epidemiológica (CVE), da Secretaria do Estado da Saúde, realizaram inquérito epidemiológico com a finalidade de caracterizar hipóteses diagnósticas, curva epidêmica e fatores de risco.

Os pacientes tiveram seu quadro clínico e laboratorial avaliado e classificado conforme os critérios vigentes para definição de infecção hospitalar (IH). Citamos abaixo a classificação utilizada:

- Casos confirmados de IH: pacientes com sinais, sintomas clínicos e alterações laboratoriais ou radiológicas compatíveis com infecção e com resultado de cultura microbiológica positivo.

- Casos compatíveis com IH: pacientes com sinais, sintomas clínicos e alterações laboratoriais ou radiológicas compatíveis com infecção, porém sem resultado positivo de cultura microbiológica.

- Casos de possivel IH: pacientes com sinais, sintomas clínicos e alterações laboratoriais compatíveis com infecção, porém compatíveis também com outras síndromes.

Avaliados pelos critérios acima, os casos se distribuíram da seguinte forma: três confirmados, três compatíveis e dois possíveis. Um caso foi excluído. Três

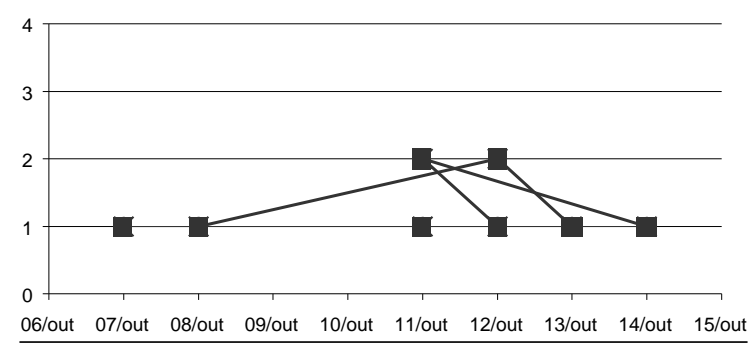

Figura 1 - Curva epidêmica, mostrando a data de início dos sintomas.

neonatos tinham quadro pneumônico. Para os demais, não foi possível a identificação de um sítio primário de infecção, sendo eles diagnosticados como infecção primária da corrente sangüínea, com ou sem confirmação laboratorial. Portanto, apenas um caso não se caracterizou como $\mathrm{IH}$, não havendo qualquer achado que corroborasse a hipótese de etiologia não infecciosa para os demais oito óbitos.

\section{Avaliação epidemiológica}

Para os oito casos de $\mathrm{IH}$, o intervalo entre o início dos sintomas e o óbito variou de um a quatro dias (mediana $=2$ ). A curva epidêmica (Figura 1) demonstrou não haver simultaneidade na ocorrência do agravo. Seu aspecto sugere epidemia propagada (pessoa a pessoa), e não de fonte comum. A densidade de incidência de IH na UTI foi avaliada através de um diagrama de controle (Figura 2). A média típica foi calculada excluindo-se o mês de outubro. Foram utilizados os critérios descritos por Sellick et al para cálculo do Limite de Alerta Superior (LAS = média típica $+2 \times$ desvio padrão) e Limite de Controle Superior $\left(\mathrm{LCS}=\right.$ média típica $+3 \mathrm{x}$ desvio padrão). ${ }^{1}$ Observando o diagrama de controle, pode-se constatar que a taxa global de IH em setembro já tangencia o LAS.

\section{Provas microbiológicas}

Culturas de espécimes clínicos identificaram Klebsiella
Correspondência para/ Correspondence to: Secretaria de Estado da Saúde de São Paulo

Centro de Vigilância Epidemiológica

Av. Dr. Arnaldo, $35112^{\circ}$ andar sala 1218

01246-901 São Paulo, SP, Brasil

E-mail: agencia@saude.sp.gov.br

Disque CVE - Central: 0800-555466
*Texto de difusão técnico-científica da Secretaria de Estado da Saúde de São Paulo, com a colaboração de técnicos do Centro de Vigilância Epidemiológica, Centro de Vigilância Sanitária e Instituto Adolpho Lutz 


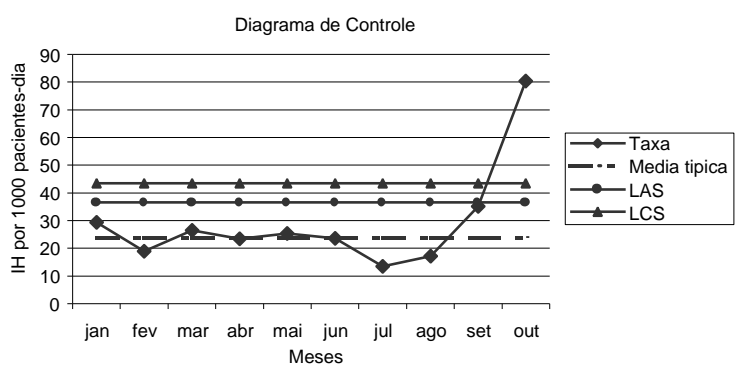

Figura 2 - Diagrama de controle da densidade de incidência de IH na UTI neonatal. (LAS = Limite de Alerta Superior; LCS = Limite de Controle Superior)

pneumoniae produtora de beta-lactamase de amplo espectro (ESBL) em sangue e ponta de cateter em dois pacientes. Um terceiro teve culturas de sangue e líquor positivas para Enterobacter cloacae. Durante a investigação, realizaram-se culturas de vigilância (swabs de nasofaringe, orofaringe, axilas e períneo) em doze pacientes que permaneciam internados no berçário, unidade de atendimento semi-intensivo. Em seis deles foi identificada Klebsiella pneumoniae $\mathrm{ESBL}(+)$, fenotipicamente idêntica àquela isolada dos casos de $\mathrm{IH}$.

\section{Fatores de risco}

Todos os pacientes haviam sido internados na UTI neonatal com doença de base grave. O período de internação variou de 2 a 44 dias (média $=17$ dias). $O$ fato de todos os neonatos da UTI terem obituado dificulta a realização de estudos de coorte retrospectiva ou casocontrole. Ainda assim, realizamos um estudo de casocontrole utilizando como controles os pacientes que estiveram internados na UTI neonatal entre 1 e 8 e aqueles que estiveram no berçário durante o mesmo período. Foram analisados oito casos e 15 controles. Os resultados demonstraram que o peso ao nascer e o período de internação não variou significativamente entre os dois grupos. Em análise univariada, associaram-se positivamente com a ocorrência de IH: ventilação mecânica, presença de catéter central, utilização de nutrição parenteral, uso de sedação, antimicrobianos, água destilada e ranitidina. Esses são os fatores de risco habitualmente observados nas infecções em berçário.

\section{CONCLUSÕES}

A avaliação clínico-epidemiológica aponta para a ocorrência de infecção hospitalar em 8 dos 9 casos. O formato da curva epidêmica sugere um surto propagado (paciente a paciente), e não de fonte comum. Não encontramos evidência de contaminação em medicações ou soluções. Por outro lado, três pacientes tiveram algum agente isolado, sendo Klebsiella pneumoniae. o mais comum.

Surtos de infecção por bacilos Gram-negativos em berçários de alto risco são bem descritos na literatura. Não controlados, esses eventos podem determinar mortalidade significativa. É digno de nota que um estudo mexicano encontrou associação entre hemoculturas positivas para Klebsiella sp. e maior risco de óbito por sepse neonatal. Sobre esse agente, é preocupante a incidência do fenótipo produtor ESBL (beta-lactamase de amplo espectro), que é resistente à maior parte dos antimicrobianos utilizados na prática clínica.

As recomendações feitas ao hospital tiveram como foco a realização de estrita vigilância epidemiológica das infecções hospitalares, com seguimento cuidadoso das taxas e otimização dos processos de controle.

\section{OS NOVOS DESAFIOS DA VIGILÂNCIA: AS CAUSAS EXTERNAS NO ESTADO DE SÃO PAULO}

Nos últimos anos, o Brasil vem experimentando progresso em alguns indicadores de saúde, tais como o aumento da expectativa de vida, a diminuição da mortalidade infantil e melhora dos indicadores de saúde bucal, entre outros. Ao mesmo tempo, novos desafios surgem na área de abrangência da saúde pública. Dentre eles, o mais importante é o crescimento das causas externas que vêm se destacando não somente no Brasil, mas em vários países do mundo. Sob essa sigla estão englobados diferentes tipos de causas, algumas classificadas como intencionais (suicídios e homicídios) e outras não intencionais (quedas, afogamentos, acidentes de trânsito, etc.). A mortalidade por estas causas atingiu proporções tão altas que trouxe reflexos na expectativa de vida da população, especialmente entre homens. Além disso, determinam uma crescente demanda aos serviços de saúde, seja em hospitalizações ou atendimentos nas emergências.

A visão tradicional de que os "acidentes" eram frutos do acaso resultou em uma certa demora da área da saúde pública em concentrar esforços para responder ao problema. Também é relativamente recente o uso das palavras "violência" e "prevenção" numa mesma sentença. Entretanto, atualmente considera-se que o impacto das causas externas pode ser reduzido, do mesmo modo que foi possível alcançar a redução das doenças infecciosas e das mortalidades infantil e materna. Acidentes podem ser previsíveis e, portanto, preveníveis, assim como fatores que contribuem para as respostas violentas (individuais ou socioeconômicos) podem ser conhecidos e mudados.

De maneira similar a qualquer outro agravo à saúde, o estabelecimento de estratégias de prevenção encontra-se diretamente relacionado com a existência de bons sistemas de informações. Portanto, os conceitos da vigilância que envolvem a prevenção e o controle das doenças devem ser aplicados a essa nova área. O Centro de Vigilância Epidemiológica "Alexandre Vranjac" (CVE), da Secretaria de Estado da Saúde de São Paulo, está estabelecendo uma área voltada 
para o acompanhamento desses agravos, cujo objetivo é a elaboração, análise e divulgação das informações, possibilitando o planejamento de propostas de controle dos acidentes e violências.

\section{A situação no Estado de São Paulo}

As informações de mortalidade aqui utilizadas são provenientes do Sistema de Informações de Mortalidade (SIM) e as de morbidade são provenientes do Sistema de Internações Hospitalares do Sistema Único de Saúde (SIH/SUS), construído a partir dos dados da Autorização de Internação Hospitalar (AIH). Esses bancos foram disponibilizados pela Fundação Seade (2002, último ano disponível). Os dados populacionais para a construção das taxas são os do Censo 2000.

Em 2002, em São Paulo, foram 32.331 as vítimas fatais de causas externas (13,2\% do total de mortes), ocupando o terceiro lugar entre as causas de morte no Estado. Porém, em algumas Direções Regionais de Saúde (DIR) já ocupam o segundo lugar. O sexo masculino é o mais atingido, fato habitualmente verificado, com coeficiente atingindo 148,8/100.000 habitantes, enquanto a taxa no sexo feminino foi de $23,6 /$ 100.000. Isso significa que o risco de um homem morrer vítima de violência é 6,2 vezes maior do que uma pessoa do sexo oposto. Dependendo do tipo de causa, esse risco pode tornar-se maior. Essas mortes atingem desproporcionalmente os adolescentes e adultos jovens, uma vez que a faixa etária entre 15 a 29 anos concentrou 43,3\% das vítimas fatais. Embora não tenha a mesma expressão em números absolutos, a faixa de 60 anos e mais também exibe coeficientes altos, apontando que devem ser desenvolvidas ações voltadas especificamente para esse grupo.

Em relação aos tipos de causas externas, os homicídios lideraram com $45,9 \%$ do total de mortes (coeficiente de 38,9/100.000). No entanto, isso não é observado em todas as Direções Regionais de Saúde (DIRs), localizando-se naquelas que compõem a Região Metropolitana de São Paulo e outros grandes centros urbanos do Interior. É nesse tipo de causa que se verifica o maior diferencial nas taxas segundo os sexos. Os acidentes de transporte foram responsáveis por $21,2 \%$ do total de mortes por essas causas (coeficiente de 17,9/100.000). $\mathrm{O}$ percentual de óbitos cuja intenção foi indeterminada, $12 \%$, aponta a necessidade de medidas para melhorar a qualidade da informação no Estado.

Em decorrência dessas causas, 172.951 pessoas foram internadas no Sistema Único de Saúde $(7,4 \%$ do total), o que determinou um custo de um pouco mais de R\$ 109 milhões, ou 8,2\% do gasto total. Essa representação percentual das causas externas um pouco maior nos gastos que no número de internações indicam que devem ser realizados estudos para esclarecer se essas causas determinam hospitalizações mais dispendiosas.
Enquanto entre as vítimas fatais o componente intencional prepondera, nas internações é o componente não intencional o que apresenta maiores percentuais, com destaque para as quedas.

\section{Perspectivas}

Pelos dados apresentados é possível observar que as causas externas se constituem numa importante demanda para a área da saúde no Estado de São Paulo.

Muitas e diversas estratégias vêm se mostrando efetivas por todo o mundo na prevenção dos acidentes de transporte, das quedas entre idosos, intoxicações/envenenamentos em crianças, entre outros eventos Já, em relação às violências, é preciso reconhecer que o seu enfrentamento não é tarefa simples e as intervenções devem envolver os vários setores da sociedade.

O propósito do Centro de Vigilância Epidemiológica é produzir conhecimento para subsidiar políticas públicas de prevenção e controle dos acidentes e violências. Diante disso, são consideradas atividades prioritárias:

- análise rotineira da morbi-mortalidade por causas externas, a partir das informações das fontes oficiais disponíveis (SIM e SIH).

- elaboração de um modelo de vigilância epidemiológica para as causas externas, baseado na integração de diversas fontes de dados, uma vez que os registros realizados na área da saúde informam satisfatoriamente acerca do perfil das vítimas, mas carecem de informações sobre as circunstâncias do evento e outras características essenciais para as políticas de prevenção e controle. Dada a magnitude e transcendência, esse modelo será iniciado com os homicídios. Algumas parcerias já foram estabelecidas com universidades e outras secretarias do Governo do Estado de São Paulo.

- análise das informações provenientes das notificações de maus tratos na infância e adolescência, encaminhadas pelos municípios nos quais essa atividade está implantada.

O desenvolvimento de um modelo de vigilância epidemiológica para causas externas visa avançar no conhecimento acerca dos fatores de risco em nosso meio. Essa proposta vai ao encontro da sugestão da Organização Mundial de Saúde (OMS), que preconiza a integração de informações das fontes já existentes como um caminho menos dispendioso para aumentar a conhecimento acerca do problema, uma vez que são utilizados os registros rotineiramente realizados. De acordo com isso, foi obtido junto à OMS o direito de tradução para a língua portuguesa do Injury Surveillance Guidelines, que auxiliará no estabelecimento de sistemas de vigilância de causas externas não somente no Brasil, mas também em outros países de língua portuguesa que se encontram em situação de conflito. 


\section{EPIDEMIOLOGIA DA LEISHMANIOSE VISCERAL AMERICANA}

A Leishmaniose Visceral Americana (LVA) é uma doença crônica, sistêmica, caracterizada por febre de longa duração, perda de peso, astenia, adinamia, dentre outras manifestações. Trata-se de uma antropozoonose transmitida ao ser humano pela picada do inseto contaminado. Inicialmente concentrada em áreas silvestres ou em pequenas localidades rurais, vem ocorrendo, agora, em centros urbanos de médio e grande portes. Quando não tratada, pode evoluir para óbito em um ou dois anos após o aparecimento da sintomatologia.

Considerada uma doença emergente em indivíduos portadores da infecção pelos vírus da Imunodeficiência Adquirida (HIV), a LVA tornou-se uma das doenças mais importantes da atualidade. Encontra-se entre as seis endemias consideradas prioritárias no mundo por sua ampla distribuição, principalmente na Ásia, Europa, África e Américas. No Brasil, a LVA está em franca expansão geográfica.

Na América do Sul, foi primeiramente identificada em 1913, no Paraguai. Já o primeiro caso brasileiro relatado data de 1934 nas regiões Norte e Nordeste, durante pesquisas de febre amarela. Desde então, a doença vem sendo descrita em vários municípios. Até 1980, 90\% estavam concentrados no Nordeste, considerada área de alta endemicidade. A partir de 1990, a transmissão da doença vem ocorrendo em diversas cidades de todas as regiões do Brasil, exceto a Sul, em zonas rurais, periurbanas e urbanas de grandes centros, tais como Rio de Janeiro (RJ), Belo Horizonte (MG), Araçatuba (SP) e Corumbá e Três Lagoas (MS). Atualmente, a LVA está registrada em 19 dos 27 estados brasileiros (dado em revisão - FUNASA 2003).

No Estado de São Paulo, a doença era conhecida apenas através dos casos importados, porém, em 1999 foi confirmado o primeiro caso humano autóctone no município de Araçatuba. Até dezembro de 2003 mais de 300 casos humanos foram confirmados na região Oeste do Estado, com 35 municípios classificados como de transmissão e, destes, 20 com casos humanos autóctones.

No Brasil, a doença é causada por um protozoário da família Tripanosomatidae, gênero Leishmania, espécie Leishmania chagasi. Apresenta-se em duas formas: a amastigota, obrigatoriamente intracelular em vertebrados, e a promatísgota, que se desenvolve no tubo digestivo dos vetores invertebrados e em meios de culturas artificiais.

Os reservatórios do agente etiológico, no ambiente silvestre, são as raposas (Dusycion vetulus e Cerdocyon thous) e o marsupial (Didelphis albiventris). $\mathrm{Na}$ área urbana, o cão (Canis familiaris), que apresenta intenso parasitismo cutâneo, permitindo uma fácil infecção do mosquito, determinante importante na cadeia epidemiológica. O reconhecimento das manifestações clínicas no cão é de fundamental importância para a adoção das medidas de controle da doença.

Os vetores documentados até o momento como transmissores da Leishmania (L.) chagasi, no Brasil, são insetos do gênero Lutzomya longipalpis (popularmente conhecido como palha, birigui, tatuquiras) e, recentemente, o Lutzomya cruzi, no Mato Grosso do Sul. Seu habitat é o domicílio e o peridomicílio humano. A fêmea se alimenta do sangue de mamíferos e aves.

A infecção do vetor ocorre pela ingestão, durante o repasto sangüíneo, de formas amastigotas existentes no citoplasma de macrófagos presentes na derme do hospedeiro infectado. O período de incubação é de 10 dias a 24 meses, em média de dois a quatro meses. A suscetibilidade é universal, porém, a incidência é maior em crianças. A Leishmania (L.) chagasi tem acentuado tropismo pelo sistema retículo endotelial, gerando intenso parasitismo disseminado, com o aumento do volume do fígado, baço, medula óssea e linfonodos e repleção da medula óssea, com redução da hematopoese medular, seqüestro esplênico, que em conjunto resultam em anemia, trombocitopenia e leucopenia.

Dependendo da interação agente-hospedeiro, a infecção por Leishmania chagasi e da resposta do organismo infectado, clinicamente pode se apresentar na forma assintomática ou oligoassintomática com sinais totalmente inespecíficos e resolução espontânea, a quadros agudos caracterizado por febre alta, tosse, diarréia acentuada, adinamia, discreta visceromegalia. $\mathrm{Na}$ forma clássica da doença, mais freqüente nas áreas endêmicas, os sintomas podem ter evolução prolongada com sinais de desnutrição proteico-calórica e aspecto edemaciado do paciente com abdômen protuso devido à hepatoesplenomegalia, alterações de pele (coloração pardacenta ou de cera velha nas Américas e escurecida na Índia).

O diagnóstico é baseado em dados epidemiológicos, clínicos e laboratoriais. O diagnóstico diferencial é feito com a febre tifóide, tuberculose, malária, esquistossomose, AIDS, mononucleose infecciosa, hepatite crônica, cirrose, leucemia, linfoma e endocardite bacteriana.

No atual estágio de conhecimento, o Programa de Controle da LVA, da Secretaria de Estado da Saúde, tem o objetivo de reduzir as taxas de letalidade e morbidade em humanos, por meio de diagnóstico e tratamento precoce, e diminuir os riscos de transmissão da doença, mediante controle da população de reservatórios e do agente transmissor. Também está prevista a educação em saúde para que as populações residentes em áreas endêmicas possam, uma vez informadas, adotar medidas que auxiliem na preservação do meio ambiente e, conseqüentemente, na diminuição dos riscos de transmissão da infecção. 